\title{
Prevención de trombosis protésica con anticoagulación luego de sustitución valvular aórtica con bioprótesis. Metaanálisis
}

\author{
Bres. Julia Tabó1, Valentina Resquin¹, Carolina Silveira¹, Analía Souza1, \\ Leticia Umpiérrez¹, Romina Urrutia1, Dr. Víctor Dayan²
}

\begin{abstract}
Resumen
Antecedentes: se ha reportado mayor riesgo de trombosis y eventos clínicos en pacientes que reciben bioprótesis luego de sustitución quirúrgica de la válvula aórtica, o del implante transcatéter (TAVI).

Objetivo: evaluar si el uso de anticoagulación oral (ACO), como mínimo por 90 días, disminuye o previene la aparición de eventos clínicos tromboembólicos o trombosis subclínica, en pacientes con sustitución valvular aórtica biológica. Evaluar el sangrado y mortalidad asociados con esta estrategia.

Método: se efectuó una búsqueda de artículos en bases de datos PubMed, Cochrane Central, Biblioteca Cochrane y SciELO. Los términos utilizados fueron: "Anticoagulation AND aortic bioprosthesis" y "Anticoagulation AND TAVI OR TAV”. Se establecieron criterios de inclusión y exclusión, y se realizó el análisis de eventos clínicos y subclínicos por separado. El estadístico utilizado fue odds ratio (OR). Se usó un modelo de efectos randomizados para calcular el estadístico y su intervalo de confianza (IC) de $95 \%$. Los resultados del metaanálisis se presentaron como diagrama de bosque. Consideramos significativa una $\mathrm{p}<0,05$.

Resultados: se identificaron 233 artículos; seleccionando ocho para trombosis clínica y cinco para trombosis subclínica. No se evidenció diferencia significativa en la incidencia de eventos embólicos con el uso de ACO (OR=1,01; IC95\%:

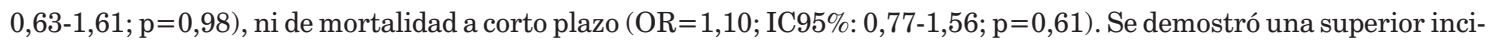
dencia de sangrados mayores en pacientes que recibieron ACO (OR=1,60; IC95\%: 1,04-2,48; p=0,03). El riesgo de trombosis subclínica protésica fue mayor en pacientes que no recibieron ACO (OR=5,52; IC95\%: 3,37-9,05; p=0,001).

Conclusiones: la ACO se asocia a un menor riesgo de trombosis protésica subclínica aumentando el riesgo de sangrado. En los grupos comparados no existe diferencia en mortalidad o fenómenos embólicos a mediano plazo.

Palabras clave: REEMPLAZO DE LA VÁLVULA AÓRTICA TRANSCATÉTER

BIOPRÓTESIS

TROMBOSIS

ANTICOAGULANTES
\end{abstract}

\section{Prevention of prosthetic thrombosis with anticoagulation after aortic valve replacement with bioprosthesis. Meta-analysis}

\section{Summary}

Background: increased risk of thrombosis and clinical events has been reported in patients receiving bioprostheses after aortic valve replacement or transcatheter aortic valve implant.

Objective: to assess whether the use of anticoagulation for at least 90 days decreases and / or prevents occurrence of thrombo-embolic or subclinical thrombosis in patients with biological aortic valve replacement. To evaluate the incidence of bleeding and mortality associated with this strategy.

1. Facultad de Medicina de la Universidad de la República. Montevideo, Uruguay.

2. Departamento de Cirugía Cardíaca del Centro Cardiovascular Universitario, Hospital de Clínicas. Montevideo, Uruguay.

Correspondencia: Dr. Víctor Dayan. 26 de Marzo 3459/602. Montevideo, Uruguay.

Correo electrónico: victor_dayan@hotmail.com

Los autores declaran no presentar conflictos de intereses.

El presente estudio no tiene fuentes de financiamiento. 
Methods: we searched articles in databases: PubMed, Central Cochrane, Cochrane Library and SciELO. The terms used were: "Anticoagulation AND aortic bioprosthesis" and "Anticoagulation AND TAVI OR TAVR". Inclusion and exclusion criteria were established, performing the analysis of clinical and subclinical events separately. The statistic used was odds ratio (OR). A randomized effects model was used to calculate the statistic and its $95 \%$ confidence interval (CI). The meta-analysis results were presented as a forest diagram. We consider $\mathrm{p}<0.05$ significant.

Results: 233 articles were identified; selecting 8 for clinical thrombosis and 5 for subclinical thrombosis. There was no significant difference in the incidence of embolic events with the use of oral anticoagulation $(\mathrm{OR}=1.01 ; 95 \% \mathrm{CI}$ : $0.63-1.61 ; \mathrm{p}=0.98)$ or short-term mortality $(\mathrm{OR}=1.10 ; 95 \% \mathrm{CI}: 0.77-1.56 ; \mathrm{p}=0.61)$. A higher incidence of major bleeding was demonstrated in patients receiving oral anticoagulation $(\mathrm{OR}=1.60 ; 95 \% \mathrm{CI}: 1.04-2.48 ; \mathrm{p}=0.03)$. The risk of subclinical prosthetic thrombosis was higher in patients who did not receive oral anticoagulation $(\mathrm{OR}=5.52$; $95 \% \mathrm{CI}$ : 3.37-9.05; $\mathrm{p}=0.001$ ).

Conclusions: oral anticoagulation is associated with a lower risk of subclinical prosthetic thrombosis, increasing the risk of bleeding. Between the groups compared, there is no difference in mortality or embolic phenomena at medium term.

Key words: $\quad$ TRANSCATHETER AORTIC VALVE REPLACEMENT

BIOPROSTHESIS

THROMBOSIS

ANTICOAGULANTS

\section{Prevenção de trombose protética com anticoagulação após troca valvar aórtica por bioprótese. Meta-análise}

\section{Resumo}

Antecedentes: foi relatado maior risco de trombose e eventos clínicos em pacientes que receberam biopróteses após substituição cirúrgica ou implante transcateter.

Objetivo: avaliar se o uso de anticoagulação por pelo menos 90 dias diminui e / ou impede a ocorrência de eventos clínicos tromboembólicos ou subclínicos em pacientes com substituição da válvula aórtica biológica. Avaliar a incidência de sangramento e mortalidade associados a essa estratégia.

Métodos: pesquisamos artigos nas bases de dados: PubMed, Central Cochrane, Cochrane Library e SciELO. Os termos utilizados foram: "Anticoagulation AND aortic bioprosthesis" e "Anticoagulation AND TAVI OR TAVR". Os critérios de inclusão e exclusão foram estabelecidos, realizando a análise dos eventos clínicos e subclínicos separadamente. A estatística utilizada foi odds ratio (OR). Um modelo de efeitos randomizados foi utilizado para calcular a estatística e seu intervalo de confiança de 95\% (IC). Os resultados da metanálise foram apresentados como um diagrama florestal. Consideramos $\mathrm{p}<0,05$ significativo.

Resultados: foram identificados 233 artigos; selecionando 8 para trombose clínica e 5 para trombose subclínica. Não houve diferença significativa na incidência de eventos embólicos com o uso de ACO (OR = 1,01; IC95\%: 0,63-1,61; p = 0,98) ou mortalidade a curto prazo (OR = 1,10; IC95\%: 0,77-1,56; $\mathrm{p}=0,61)$. Uma incidência maior de sangramento maior foi de-

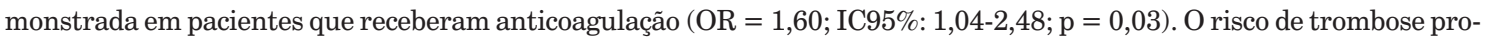
tética subclínica foi maior nos pacientes que não receberam anticoagulação (OR = 5,52; IC 95\%: 3,37-9,05; $\mathrm{p}=0,001)$.

Conclusões: a anticoagulação oral está associada a um menor risco de trombose protética subclínica, aumentando o risco de sangramento. Nos grupos comparados, não há diferença na mortalidade ou no fenômeno embólico a médio prazo.

Palavras chave: $\quad$ SUBSTITUIÇÃO DA VALVA AÓRTICA TRANSCATETER

BIOPRÓTESE

TROMBOSE

ANTICOAGULANTES

¿Qué aporta este estudio al conocimiento actual?

El uso de anticoagulación en pacientes con bioprótesis aórtica se asocia a menor riesgo de trombosis subclínica. Sin embargo, el procedimiento acarrea mayor riesgo de sangrado sin afectar la mortalidad, ni el riesgo embólico. Por lo tanto, hasta que se definan las consecuencias de la trombosis subclínica en la evolución a largo plazo, la anticoagulación debería reservarse a pacientes con bajo riesgo de sangrado.

\section{Introducción}

La incidencia de la enfermedad valvular aórtica de causa degenerativa ha ido aumentando en las últimas décadas debido al incremento de la esperanza de vida de la población, lo que representa un gran reto a nivel sanitario, social y económico ${ }^{(1)}$. La sustitución valvular aórtica (SVA) es el tratamiento curativo para esta enfermedad y es una de las intervenciones quirúrgicas más frecuentes en los centros de cirugía cardíaca a nivel mundial. Para la SVA pueden utilizarse bioprótesis o válvulas mecánicas y su elección dependerá de varios factores, 
dentro de los cuales se encuentran la edad y el terreno del paciente ${ }^{(1)}$. En los últimos años ha surgido la SVA transcatéter (TAVI) de la válvula aórtica, reservada inicialmente para pacientes de alto riesgo.

Dentro de las ventajas de la utilización de las bioprótesis destacan su menor riesgo trombogénico en comparación con las válvulas mecánicas y el no requerimiento de tratamiento antitrombótico de por vida. Como desventaja, presentan menor durabilidad $^{(2)}$.

La trombosis de una válvula biológica es infrecuente, con una incidencia anual de 0,01\%-1,26\%. Representa una complicación grave que puede llevar a la muerte o generar secuelas permanentes que disminuyen la calidad de vida ${ }^{(3)}$. El riesgo de experimentar eventos tromboembólicos es mayor durante los 90 días posteriores a la SVA, tiempo que se considera necesario para la endotelización del implante ${ }^{(2)}$.

En cuanto a la trombosis subclínica de la válvula bioprotésica, se puede valorar mediante estudios imagenológicos, como la tomografía computarizada (TC) de cuatro dimensiones, siendo este el método de imagen más sensible para detectar la forma de apertura/cierre limitado de la válvula y el engrosamiento de esta ${ }^{(4)}$. Otro estudio de imagen complementario es la ecocardiografía, que puede apreciar hallazgos compatibles con trombosis subclínica como el aumento de los gradientes transaórticos, reducción del área de la válvula aórtica $<1,2 \mathrm{~cm}^{2}$ y la movilidad anormal de la cúspide, que predicen la presencia de trombosis valvular ${ }^{(5)}$.

Actualmente existe evidencia que relaciona la trombosis subclínica de la válvula bioprotésica con el deterioro hemodinámico valvular prematuro y la degeneración estructural de la válvula ${ }^{(6)}$. Además, la trombosis subclínica de la valva puede constituir un factor de riesgo para eventos cerebrales subagudos u otros eventos tromboembólicos ${ }^{(6)}$.

Las guías actuales de la Sociedad Europea de Cardiología (ESC) y la European Association for Cardio-Thoracic Surgery (EACTS) recomiendan considerar la anticoagulación oral (ACO) durante los primeros 90 días del posoperatorio de SVA, con una recomendación $\mathrm{IIb}$, nivel de evidencia $\mathrm{C}^{(7)}$. Las guías 2017 de la American Heart Association/American College of Cardiology/American College of Chest Physicians recomiendan su utilización por al menos tres a seis meses pos-SVA con una recomendación IIa, nivel de evidencia B-NR ${ }^{(8)}$.

A pesar de estas recomendaciones, existe un debate constante acerca de los beneficios y los riesgos de la utilización de ACO. Muchos profesionales no se adhieren a estas recomendaciones y cuestionan su utilización en ausencia de otras patologías tromboembólicas, como fibrilación auricular, estados de hipercoagulación, tromboembolia venosa, etcétera, con la justificación de que existe falta de estudios clínicos que demuestren su beneficio ${ }^{(8)}$.

El objetivo de nuestro trabajo es realizar un metaanálisis para evaluar si el uso de ACO por un mínimo de 90 días disminuye o previene la aparición de eventos trombóticos o trombosis subclínica en pacientes con SVA quirúrgica biológica o TAVI, así como evaluar el riesgo de sangrado, fenómenos embólicos y mortalidad

\section{Material y métodos}

El presente estudio muestra los resultados de una revisión sistemática con metaanálisis. Se efectuó una búsqueda de artículos en bases de datos electrónicas: PubMed, Cochrane Central, Biblioteca Cochrane y SciELO utilizando términos MEsH y conectores booleanos. Los términos de búsqueda fueron los siguientes: "Anticoagulation AND bioprosthetic aortic valves" y "Anticoagulation AND TAVI OR TAVR". La búsqueda contuvo todos los estudios publicados hasta la fecha, sin restricción de idioma. Se evaluaron artículos relacionados durante la búsqueda, así como también las referencias de las revisiones del tema.

El metaanálisis incluyó: 1) Ensayos clínicos controlados aleatorizados, estudios prospectivos y retrospectivos. 2) Población: pacientes con SVA con prótesis biológica a través de TAVI o SVA quirúrgica. 3) Intervención: administración de ACO con warfarina o nuevos anticoagulantes orales (NACO) al menos por 90 días luego de la cirugía. 4) Intervención comparativa: sin administración de anticoagulación o administración de simple o doble terapia antiplaquetaria. 5) Variables resultado: presencia o ausencia de eventos trombóticos, tanto clínicos como subclínicos. Se tomaron en cuenta estudios donde se observó la presencia de elementos clínicamente objetivables luego de la sustitución valvular, tales como accidentes cerebrovasculares (ACV), accidentes isquémicos transitorios (AIT) y trombosis clínica de la prótesis biológica, y estudios en los que se evaluaron hallazgos imagenológicos de trombosis de bioprótesis subclínica, definiéndola como el engrosamiento de los velos, la disminución en la movilidad de estos o aumento de $>10 \mathrm{mmHg}$ en el gradiente transprotésico ${ }^{(9-12)}$.

Para el análisis de los eventos clínicos se excluyeron aquellos trabajos que: a) no cumplieran con los criterios de inclusión previamente mencionados; b) incluyeran a pacientes que tuvieran indicación de anticoagulación por otra patología previa a la cirugía; c) los pacientes fueran sometidos a susti- 
tución valvular mitral, y d) grupos con menos de 10 pacientes.

En la evaluación de trombosis valvular subclínica se utilizaron los mismos criterios de inclusión previamente mencionados, incluyendo, además, pacientes con anticoagulación por otra causa previa a la realización del estudio. Comparte los mismos criterios de exclusión que el análisis previo, a excepción de los trabajos en los cuales los pacientes fueron sometidos a doble sustitución valvular independientemente del tipo de procedimiento, o sustitución de válvula mitral.

La selección de artículos se realizó en etapas por dos grupos independientes de estudiantes. En cada etapa se realizó un análisis exhaustivo de artículos y se seleccionaron aquellos que cumplían con los criterios de inclusión, posteriormente se debatió entre los grupos la selección final.

\section{Estadística}

Los estadísticos de resumen utilizados fueron odds ratio $(\mathrm{OR})$ para las variables categóricas. Según la heterogeneidad de los pacientes incluidos, se utilizó un modelo de efectos randomizados o fijo para poder calcular el estadístico de resumen y su intervalo de confianza (IC) de 95\%, el cual se basó en I2. Los resultados del metaanálisis se presentaron como diagrama de bosque. El análisis fue realizado usando Review Manager Versión 5.3 (The Cochrane Collaboration, Update Software, Oxford).

\section{Resultados}

Mediante la búsqueda sistemática inicial se identificaron 233 artículos. De estos, se excluyeron 96 luego de evaluar el título. De los restantes, se analizó el resumen y se descartaron 113 artículos. Los motivos por los cuales se descartaron estos artículos se encuentran especificados en la figura 1. Se revisaron a texto completo 24 trabajos, de los cuales se seleccionaron ocho para el análisis clínico y cinco para la evaluación de trombosis protésica.

\section{Análisis de eventos clínicos}

En la tabla 1 se muestran las características de los estudios incluidos. Los mismos fueron observacionales retrospectivos ${ }^{(13-15)}$ y prospectivos ${ }^{(16,17)}$, incluyendo ensayos clínicos aleatorizados ${ }^{(18-20)}$. Los estudios incluyeron un total de 22.875 pacientes, de los cuales 13.248 no recibieron anticoagulación y 9.627 sí la recibieron. De los pacientes incluidos, 12.075 recibieron cirugía de revascularización coronaria (CRM), además de la SVA.

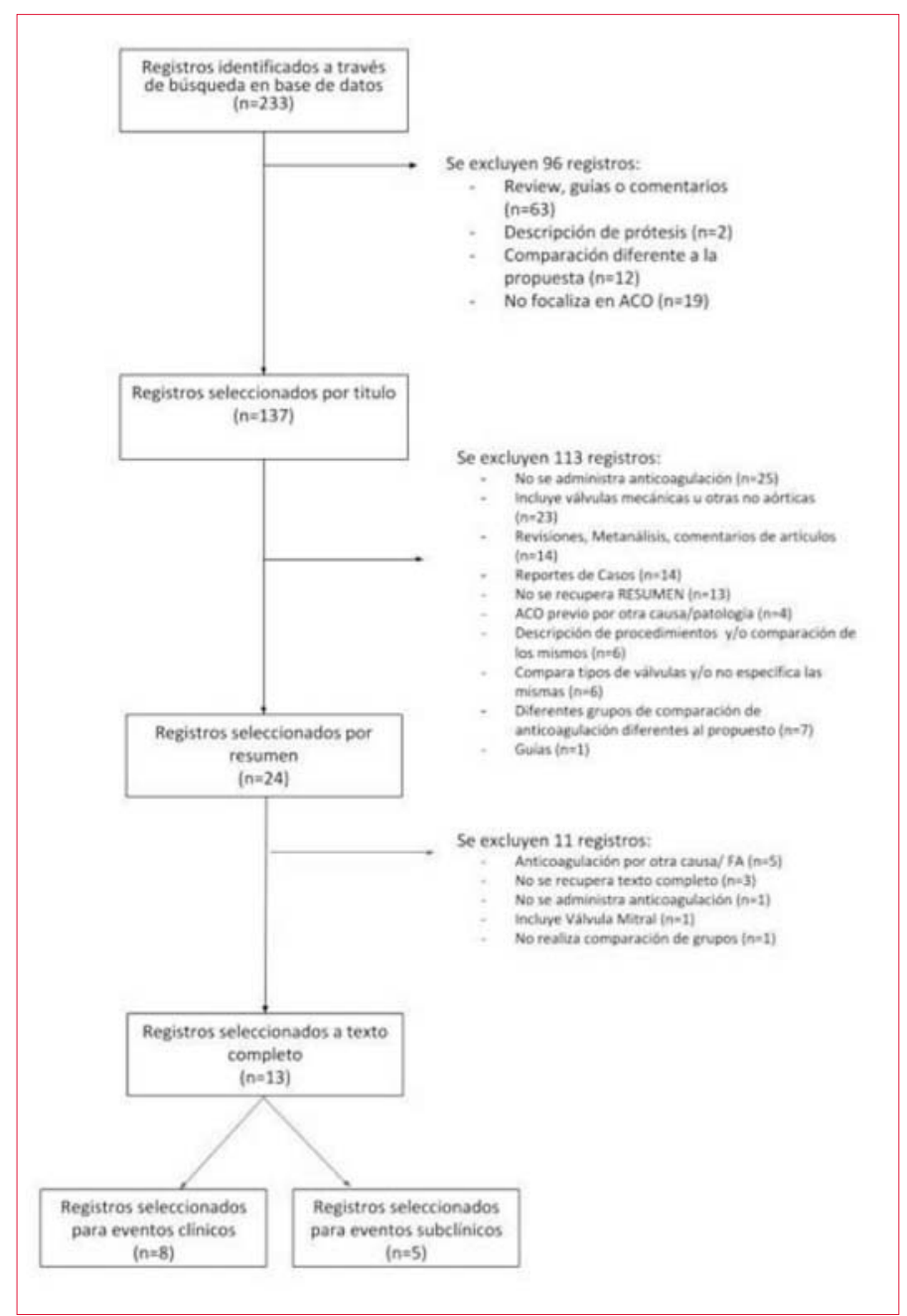

Figura 1. Diagrama de flujo del proceso de inclusión de los trabajos.

\section{Embolia}

Todos los trabajos aportaron datos de eventos embólicos. A partir de los resultados obtenidos de los ocho estudios, la incidencia de eventos embólicos osciló entre $1 \%^{(13)}$ y $5 \%^{(14)}$ para el grupo de anticoagulados, y entre $0 \%^{(14)}$ y $7 \%{ }^{(13)}$ para los no anticoagulados. El análisis global demostró que no existe diferencia significativa en la incidencia de eventos embólicos entre ambos grupos (OR=1,01; IC95\%: $0,63-1,61 ; p=0,98)$. El grado de heterogeneidad fue bajo con un $\mathrm{I} 2=0 \%$, por lo que utilizamos un modelo de efectos fijo. Ninguno de los estudios seleccionados mostró resultados estadísticamente significativos para esta variable (figura 2).

\section{Sangrado}

El sangrado fue una variable secundaria a estudiar relacionada con el uso de la terapia antitrombótica. La incidencia de sangrados mayores osciló para el grupo de warfarina entre $0 \%{ }^{(16)}$ y $8,8 \%{ }^{(19)}$, en com- 
Tabla 1. Características de los estudios incluidos para la evaluación clínica.

\begin{tabular}{|c|c|c|c|c|c|c|c|}
\hline & \multicolumn{2}{|c|}{ Carasterititicas del estudio } & \multicolumn{3}{|c|}{ 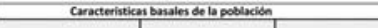 } & \multirow[b]{2}{*}{ 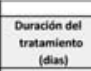 } & \multirow[b]{2}{*}{$\begin{array}{c}\text { Tiempo de } \\
\text { seguimiento } \\
\text { (meses) }\end{array}$} \\
\hline Autores & AnO & Diseto del Estudio & Edad & ระท & MniA & & \\
\hline Rafia S, y col. & 2016 & $\begin{array}{l}\text { Emaryo cinico } \\
\text { randomirado, unictemtrico }\end{array}$ & $\begin{array}{c}\text { Wartarina: } 7.18 \\
6.4\end{array}$ & $\begin{array}{c}\text { Wartavina: } 51.42 \\
125\end{array}$ & $\begin{array}{l}\text { Warfarina (H-W):2 } \\
\text { Aas (H-M):2 }\end{array}$ & $\infty$ & 3 \\
\hline Oserti T.yeal. & 2004 & $\begin{array}{l}\text { Observacional } \\
\text { Prospection }\end{array}$ & $\begin{array}{c}\text { Wartarina: } 72.92 \\
7.1 \\
\text { Mas: } 70.0=8.8\end{array}$ & $\begin{array}{c}\text { Wartwine } 55.6 \mathrm{z} \\
\text { 13.6 AS: } 57.1 \mathrm{t} \\
13.6\end{array}$ & 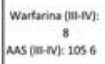 & $\infty$ & 3 \\
\hline Elbardissi A, ycol. & 2010 & 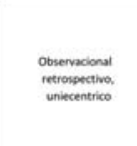 & 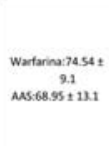 & $\begin{array}{l}\text { Wartarina } 54: 13 \\
\text { AAS: } 58: 11\end{array}$ & 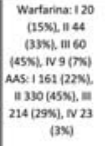 & 90 & 3 \\
\hline colli $\begin{array}{c}\text { yed. } \\
\text {. }\end{array}$ & 2007 & $\begin{array}{l}\text { Ensaro cinico } \\
\text { randomirado, } \\
\text { multictentico }\end{array}$ & $\begin{array}{c}\text { Wutarinu } 69.5 \\
\pm 3.3 \\
\text { ads: } 70.7 \pm 3.7\end{array}$ & 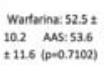 & 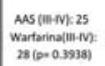 & 90 & 3 \\
\hline $\begin{array}{l}\text { Arennan IMy } \\
\text { colisboradores }\end{array}$ & 2012 & $\begin{array}{l}\text { Observacional } \\
\text { Retrospectivo, } \\
\text { multicenterico }\end{array}$ & 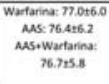 & $\begin{array}{c}\text { MS } 54: 13 \\
\text { Wartarina Sst14 } \\
\text { Wartarino+aAs } \\
\text { S3:13 }\end{array}$ & - & $\infty$ & 3 \\
\hline Of Marco y col & 2007 & $\begin{array}{l}\text { Observacional } \\
\text { pronpectiva, unicentrica. }\end{array}$ & $\begin{array}{l}\text { Wartarina 7s:5 } \\
\text { Aas: } 1: 75=6\end{array}$ & - & - & 90 & $24: 14$ \\
\hline $\begin{array}{l}\text { Acever Velisquez } \\
\text { yeol }\end{array}$ & 2017 & $\begin{array}{l}\text { Observacional Retrospec } \\
\text { tivo,unicentrica. }\end{array}$ & 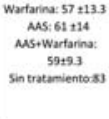 & 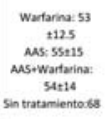 & - & 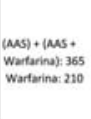 & 12 \\
\hline A-Atasi yed & 2012 & $\begin{array}{l}\text { Insarpo clinico abierta, } \\
\text { randomisado. }\end{array}$ & $\begin{array}{c}\text { Mas } 72 \pm 9 \\
\text { Aas } 5 \text { Wartarina: } \\
71 \pm 10\end{array}$ & - & - & $\infty$ & 12 \\
\hline
\end{tabular}

AAS: ácido acetilsalicílico.

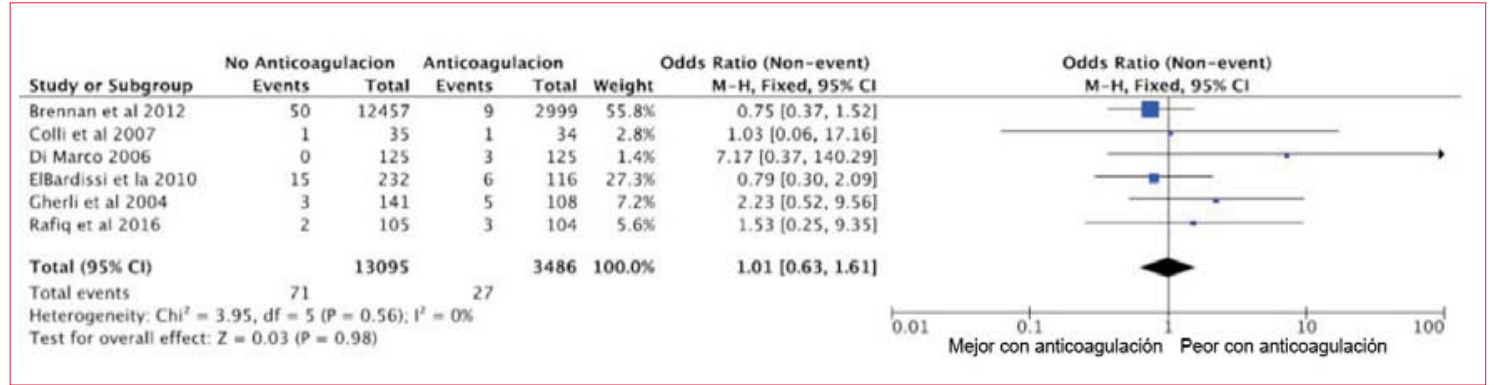

Figura 2. Diagrama de bosque relacionado con las embolias sistémicas.

paración con el grupo control que osciló entre $0 \%(16)$ y $3,5 \%(14)$. El análisis global demostró que existe una superior incidencia de sangrado mayor en pacientes que recibieron tratamiento antitrombótico $(\mathrm{OR}=1,60 ; \mathrm{IC} 95 \%: 1,04-2,48 ; \mathrm{p}=0,03)$. El grado de heterogeneidad en este caso también fue bajo con un $\mathrm{I} 2=0 \%$, y se utilizó un modelo de efectos fijo (figura 3$)$.

\section{Mortalidad}

La mayor parte de las defunciones ocurrió luego de los 90 días posteriores a la cirugía $(13,14,17,19,20)$. En tres de los trabajos incluidos, el seguimiento fue mayor $^{(15,16,18)}$. El mayor seguimiento tuvo una media de 24 meses $\pm 14^{(16)}$. La incidencia de muerte en el grupo de warfarina osciló entre $0 \%{ }^{(15,18)}$ y $5,9 \%{ }^{(19)}$, siendo para el grupo control entre $0 \%{ }^{(15,18)}$ y $5,7 \%$
(19). En este caso, el grado de heterogeneidad de los resultados fue moderado ( $\mathrm{I} 2=56 \%$ ), por lo cual se utilizó un modelo de efectos randomizados. Encontramos que no existe diferencia significativa en la mortalidad a corto plazo entre los pacientes que recibieron terapia antitrombótica y quienes no la recibieron $(\mathrm{OR}=1,10$; IC95\%: 0,77-1,56; $\mathrm{p}=0,61)$. El estudio de Aceves-Velázquez y colaboradores ${ }^{(15)}$, junto con Al-Atassi y colaboradores ${ }^{(18)}$, no reportaron dicha variable, por lo que no fueron incluidos en el análisis estadístico (figura 4).

\section{Análisis de trombosis protésica}

En la tabla 2 se muestran las características de los estudios incluidos. Se seleccionaron un total de cinco estudios, cuatro fueron observacionales retrospectivos $^{(6,10-12)}$ y uno prospectivo ${ }^{(9)}$. Los estudios in- 


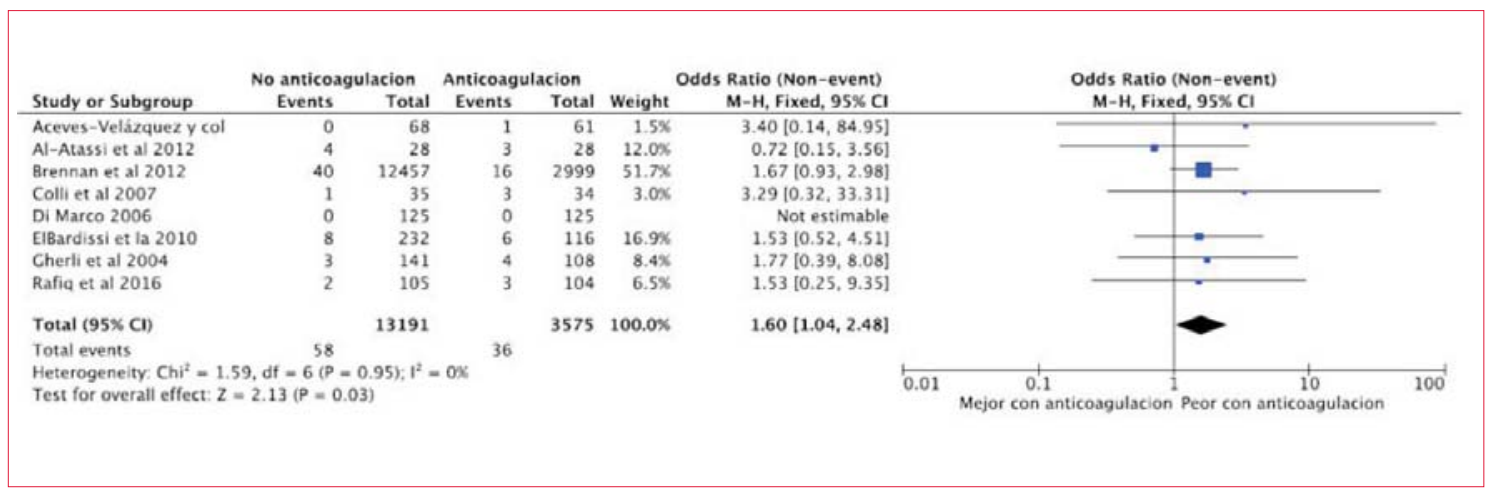

Figura 3. Diagrama de bosque relacionado con los sangrados mayores.

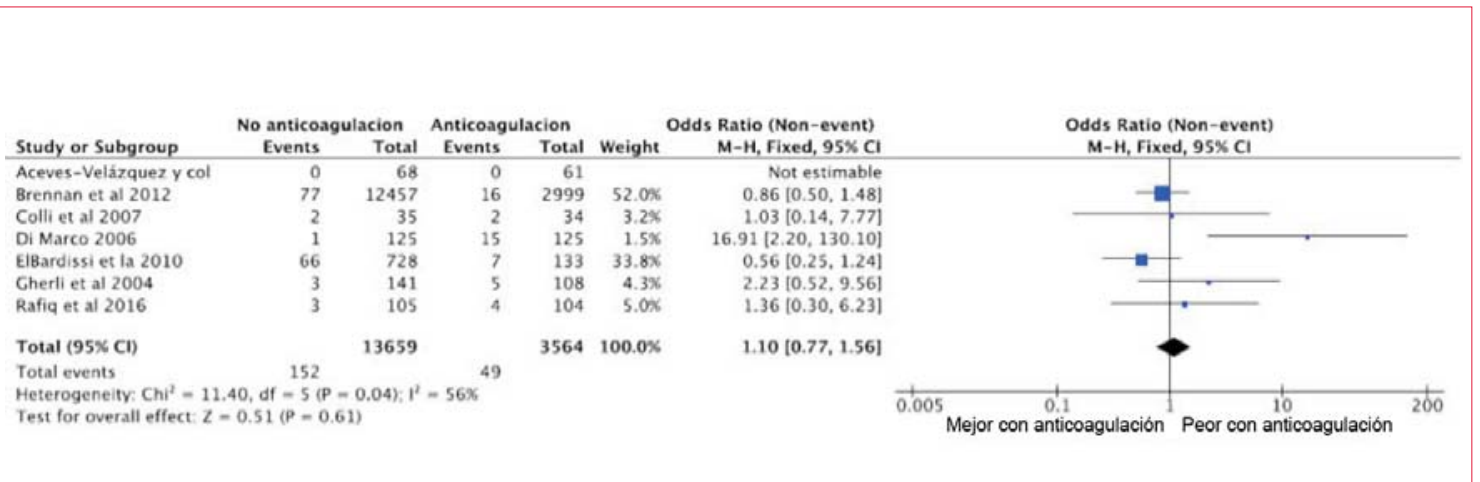

Figura 4. Diagrama de bosque relacionado con la mortalidad.

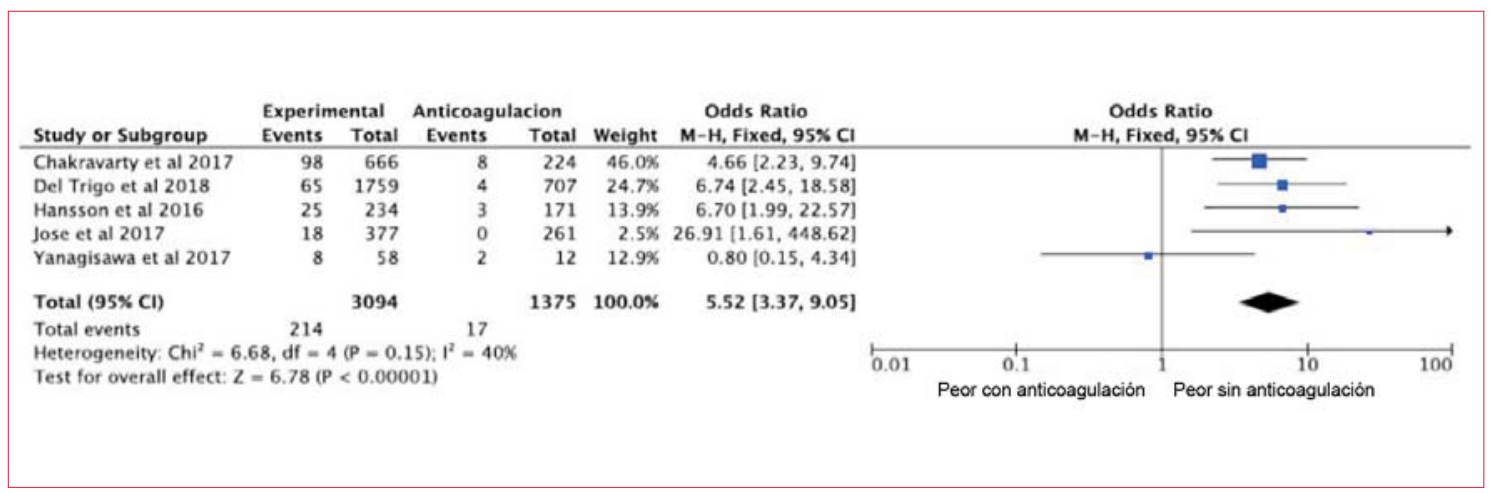

Figura 5. Diagrama de bosque relacionado con la trombosis subclínica.

cluyeron un total de 4.469 pacientes, de los cuales 4.331 recibieron TAVI y 138 SVA quirúrgica.

Dado que la mayoría de los pacientes que surgen del análisis recibieron TAVI, los resultados expresan principalmente la incidencia de trombosis protésica luego de dicho procedimiento. El grado de heterogeneidad fue moderado ( $\mathrm{I} 2=40 \%$ ), por lo cual el análisis se realizó mediante un modelo de efectos randomizados. El no uso de ACO demostró aumentar significativamente el riesgo de trombosis protésica (OR=5;52; IC95\%: 3,37-9,05) (figura 5).

\section{Discusión}

El metaanálisis realizado evidencia que el uso de ACO en pacientes que recibieron SVA está asociado a un menor riesgo de trombosis protésica a pesar de demostrar un mayor riesgo de sangrado. Asimismo, no hallamos evidencias estadísticamente significativas en el uso de ACO para prevenir la aparición de eventos trombóticos clínicamente objetivables. Estos hallazgos se contraponen con las recomendaciones actuales brindadas por las guías internacionales para el manejo de estos pacientes. 
Tabla 2. Características de los estudios incluidos relacionados con la trombosis subclínica.

\begin{tabular}{|c|c|c|c|c|c|}
\hline Autores & Año & $\begin{array}{l}\text { Diseño del } \\
\text { Estudio }\end{array}$ & $\begin{array}{l}\text { Definición de } \\
\text { trombosis }\end{array}$ & $\begin{array}{c}\text { Tipo de } \\
\text { anticoagulación }\end{array}$ & $\begin{array}{l}\text { Tiempo de } \\
\text { seguimiento } \\
\text { (meses) }\end{array}$ \\
\hline Del Trigo & 2018 & $\begin{array}{l}\text { Observacional, } \\
\text { retrospectivo, } \\
\text { multicéntrico }\end{array}$ & $\begin{array}{c}\text { Aumento del } \\
\text { gradiente } \geq 10 \\
\mathrm{mmHg}\end{array}$ & $\begin{array}{l}\text { Warfarina y } \\
\text { NACO }\end{array}$ & 12 \\
\hline Chakravarty & 2017 & $\begin{array}{l}\text { Observacional } \\
\text { Retrospectivo } \\
\text { Multicentrico }\end{array}$ & $\begin{array}{c}\text { Según } \\
\text { movilidad de los } \\
\text { velos }\end{array}$ & NACO & 3 \\
\hline Jose & 2017 & $\begin{array}{c}\text { Observacional } \\
\text { retrospectivo, } \\
\text { unicentrico }\end{array}$ & $\begin{array}{c}\text { Gradiente } \\
\text { medio } \geq 20 \\
\mathrm{mmHg}, \\
\text { reduccion del } \\
\text { area a }<1,2 \\
\mathrm{~cm} 2 .\end{array}$ & $\begin{array}{l}\text { Warfarina y } \\
\text { NACO }\end{array}$ & 60 \\
\hline Hansson & 2016 & $\begin{array}{l}\text { Observacional, } \\
\text { retrospectivo, } \\
\text { unicéntrico }\end{array}$ & $\begin{array}{c}\text { Engrosamiento } \\
\text { valvular difuso } \\
\text { de } 1 \text { o más } \\
\text { valvas. }\end{array}$ & & \\
\hline Yanagisawa & 2017 & $\begin{array}{l}\text { Prospectivo, } \\
\text { unicéntrico }\end{array}$ & $\begin{array}{c}\text { Engrosamiento } \\
\text { valvular }\end{array}$ & $\begin{array}{c}\text { Warfarina y } \\
\text { NACO }\end{array}$ & 12 \\
\hline
\end{tabular}

Si bien las bioprótesis valvulares aórticas se consideran menos trombogénicas en comparación a las prótesis mecánicas, existe riesgo de desarrollar eventos tromboembólicos ${ }^{(2)}$. Algunos autores consideran que la incidencia de trombosis valvular puede estar subestimada, ya que en los primeros estudios efectuados en pacientes con SVA, no estaba indicado el ecocardiograma de rutina, por lo cual la trombosis valvular no siempre era constatada ${ }^{(21)}$. El riesgo de presentar trombosis valvular y eventos tromboembólicos es mayor durante los primeros 90 días posteriores a la $\mathrm{SVA}^{(2,22)}$. Sin embargo, no existe consenso actual sobre el uso de anticoagulación luego de dicho procedimiento ${ }^{(23)}$.

La evidencia inicial en apoyo de la ACO temprana surgió de los resultados de un estudio retrospectivo unicéntrico realizado por Heras y colaboradores ${ }^{(22)}$, lo que influyó en gran parte del desarrollo de las directrices actuales. En este estudio se analizaron los pacientes que recibieron SVA mecánica o biológica, aórtica o mitral, en tres intervalos de tiempo hasta 90 días después de la cirugía. Se observó que en los primeros 10 días hubo una tasa de eventos tromboembólicos significativa en los pacientes que recibieron SVA con bioprótesis sin ACO, disminuyendo significativamente la incidencia con el transcurso del tiempo. Por lo tanto, se concluyó que la ACO temprana se debe administrar durante al menos tres meses para prevenir la apari- ción de eventos trombóticos. Este documento influyó entre los miembros de la comunidad quirúrgica a favor de la ACO y alentó a realizar más investigaciones.

De la misma forma, Moinuddeen y colaboradores $^{(24)}$, y Sundt y colaboradores ${ }^{(25)}$, concluyeron que la ACO precoz después de SVA no confiere ninguna ventaja en la prevención de eventos tromboembólicos. Los resultados de este último no identificaron diferencias significativas en las complicaciones tromboembólicas o hemorrágicas entre los dos grupos ${ }^{(25)}$. Se observó que la supervivencia y función valvular a largo plazo no se vieron afectadas negativamente al suspender la ACO temprana. No se reportaron eventos hemorrágicos debido a la administración de $\mathrm{ACO}^{(24)}$. Contrariamente a los estudios previos mencionados, Mérie y colaboradores ${ }^{(26)}$ publicaron en 2012, un estudio observacional, retrospectivo, que abarcó una población de 4.075 pacientes. Este trabajo demostró que suspender el tratamiento con warfarina durante los primeros tres meses después de la cirugía está asociado con un aumento significativo del riesgo de un evento clínico adverso, como ACV, muerte cardiovascular y complicaciones tromboembólicas. A consecuencia de este estudio, los autores cuestionan las pautas actuales sobre la duración del tratamiento antitrombótico después de SVA con prótesis biológicas. 
Un reciente metaanálisis realizado por Riaz H. y colaboradores ${ }^{(27)}$, donde se compara el uso de ACO con warfarina y la terapia antiplaquetaria, concuerda con los hallazgos obtenidos en nuestro estudio. A diferencia de nuestro trabajo, esta publicación incluyó pacientes anticoagulados por otra patología previo a la cirugía, valorando únicamente la aparición de trombosis protésica. Consideramos que la inclusión de estas variables influye en los resultados obtenidos, siendo por lo tanto, menos preciso para valorar los eventos trombóticos luego de la SVA, y así evaluar la indicación de ACO en dichos casos. Los autores concluyeron la existencia de un incremento significativo en el riesgo de sangrado, sin un efecto favorable en la prevención de eventos tromboembólicos. Por lo dicho, recomiendan la realización de estudios randomizados con el objetivo de guiar las directivas del uso de ACO en la práctica clínica diaria.

La trombosis valvular es considerada una complicación infrecuente luego de la realización de la TAVI. Esta es diagnosticada con la aparición de eventos clínicos o a través de hallazgos ecocardiográficos $^{(28)}$. La trombosis valvular subclínica fue recientemente descrita luego del advenimiento de la TC de alta resolución ${ }^{(9)}$. Las características de esta se han logrado demostrar por medio de este estudio, así como la respuesta frente a la administración de ACO para su resolución ${ }^{(28)}$.

Un conjunto de revisiones realizadas en el año 2017 por Nakatani S. ${ }^{(29)}$ y Jilaihawi H. ${ }^{(30)}$, destacan la importancia de la realización de una TC de alta resolución para detectar la trombosis valvular temprana, teniendo mayor sensibilidad que la realización de ecocardiograma transtorácico o transesofágico. La TC permite la detección de la patología en pacientes asintomáticos previo a la manifestación clínica, e incluso antes de la presencia de alteraciones hemodinámicas. La forma de presentación de la trombosis subclínica en la TC es bajo la limitación de apertura/cierre de la válvula, o el engrosamiento hipoatenuado de las valvas (HALT) ${ }^{(29)}$. La trombosis subclínica de la prótesis no tiene secuelas clínicas inmediatas, pero existe la posibilidad de manifestaciones tardías, generando complicaciones, como fenómenos embólicos o eventos clínicos.

Es por ello que se establece que la ACO puede prevenir y revertir la hipoatenuación observada y al mismo tiempo restablecer el movimiento normal de las valvas ${ }^{(28)}$. Nuestros resultados demuestran que los pacientes con ACO, luego de TAVI, presentan un riesgo significativamente menor de desarrollar trombosis subclínica.
Resta definir la relación riesgo/beneficio de dicha farmacoterapia en una población con alto riesgo de sangrado ${ }^{(29,30)}$.

\section{Limitaciones}

La amplia mayoría de los estudios incluidos en este metaanálisis corresponden a estudios observacionales, los que podrían estar sujetos a sesgo de selección, ya que la preferencia de administrar terapia antiplaquetaria, ACO o ninguna terapia, quedaba supeditada a la adherencia del médico tratante a las pautas actuales. Asimismo, también están condicionados por la presencia o ausencia de indicaciones previas para recibir ACO. Por otro lado, los regímenes antitrombóticos utilizados varían entre los diferentes trabajos, desde los grupos a comparar, a las dosis a administrar, lo cual genera variabilidad al momento de comparar los diferentes estudios.

Como se mencionó previamente, nuestros resultados demuestran el beneficio del uso de ACO en los primeros 90 días luego de la SVA para prevenir la aparición de trombosis protésica, en contraposición con el uso de terapia antiplaquetaria o sin mediar tratamiento.

Si bien la mayoría de los estudios toman como punto de partida un seguimiento mínimo de 90 días, pasado este lapso de tiempo, el seguimiento y los controles realizados por cada estudio fue diferente, generando diversidad al momento de evaluar los eventos clínicos y la trombosis valvular, requiriendo esta última un control imagenológico más riguroso. Adicionalmente hubo heterogeneidad a la hora de emplear técnicas de imagen para determinar la trombosis valvular, observando que no existen aún protocolos estandarizados que faciliten el diagnóstico precoz y oportuno de esta variable.

\section{Conclusiones}

En este metaanálisis se demostró que el uso de ACO en pacientes que recibieron SVA con TAVI o cirugía, está asociado a un menor riesgo de trombosis protésica subclínica, incrementando el riesgo de sangrado. No existió diferencia en mortalidad a mediano plazo en ambos grupos comparados. No se pudo demostrar estadísticamente el beneficio de la ACO para la prevención de eventos embólicos clínicos. Dado que los estudios incluidos para la realización de este trabajo fueron en su mayoría observacionales (retrospectivos y prospectivos), consideramos que la confirmación de nuestros resultados debe realizarse a través de ensayos clínicos aleatorizados, los que brindarían mayor evidencia científica. 
Contribución de autores

Julia Tabó, https://orcid.org/0000-0002-7331-8891 adquisición, análisis e interpretación de los datos, revisión y edición del manuscrito final. Aprobación final de la versión a ser publicada.

Valentina Resquin, https://orcid.org/0000-00021185-8585 adquisición, análisis e interpretación de los datos, revisión y edición del manuscrito final. Aprobación final de la versión a ser publicada.

Carolina Silveira, https://or-

cid.org/0000-0002-6043-2505 adquisición, análisis e interpretación de los datos, revisión y edición del manuscrito final. Aprobación final de la versión a ser publicada. Analia Souza, https://orcid.org/0000-0002-1100-7879 adquisición, análisis e interpretación de los datos, revisión y edición del manuscrito final. Aprobación final de la versión a ser publicada.

Leticia Umpiérrez. https://orcid.org/0000-

0002-0562-7019 adquisición, análisis e interpretación de los datos, revisión y edición del manuscrito final. Aprobación final de la versión a ser publicada.

Romina Urrutia, https://orcid.org/0000-0001-9276-8321 adquisición, análisis e interpretación de los datos, revisión y edición del manuscrito final. Aprobación final de la versión a ser publicada.

Víctor Dayan, https://orcid.org/ 0000-0002-5470-0585. idea original, concepción y diseño del trabajo; adquisición, análisis e interpretación de los datos, revisión y edición del manuscrito final. Comunicación con editores. Aprobación de la versión final a ser publicada.

Este artículo fue aceptado para su publicación por: Editor jefe Dr. Gerardo Soca.

\section{Bibliografía}

1. Sociedad Argentina de Cardiología. Área de Normatizaciones y Consenso. Sociedad Argentina de Gerontología y Geriatría. Valvulopatías aórticas. Rev Argent Cardiol. 2003;71(Supl 2):38-44.

2. Mydin M, Dimitrakakis G, Younis J, Nowell J, Athanasiou T, Kourliouros A. Optimal thromboprophylaxis following bioprosthetic aortic valve replacement: still a matter of debate? Interact Cardiovasc Thorac Surg. 2012; 15(1):109-14. doi: 10.1093/ icvts/ivs102

3. Cordoba-Soriano J, Puri R, Amat-Santos I, Ribeiro H, Abdul-Jawad O, del Trigo M, et al. Revisión sistemática de la trombosis protésica tras implante percutáneo de válvula aórtica. Rev Esp Cardiol. 2015;68(3):198-204. doi: 10.1016/j.recesp.2014. 10.004

4. Zhao Z, Wang M, Jilaihawi H. Imaging evaluation for the detection of leaflet thrombosis after transcat- heter aortic valve replacement. Interv Cardiol Clin. 2018; 7(3):293-9. doi: 10.1016/j.iccl.2018.03.007

5. Kappetein A, Head S, Généreux $\mathbf{P}$, Piazza $\mathbf{N}$, van Mieghem N, Blackstone E, et al. Updated standardized endpoint definitions for transcatheter aortic valve implantation: the Valve Academic Research Consortium-2 consensus document. Eur Heart J. 2012; 33(19):2403-18. doi: 10.1093/eurheartj/ehs 255

6. Chakravarty T, Søndergaard L, Friedman J, De Backer O, Berman D, Kofoed K, et al. Subclinical leaflet thrombosis in surgical and transcatheter bioprosthetic aortic valves: an observational study. Lancet 2017; 389(10087):2383-92. doi: 10.1016/ S0140-6736(17)30757-2

7. Evangelista A. Comentarios a la guía ESC/EACTS 2017 sobre el tratamiento de las valvulopatías. Rev Esp Cardiol. 2018; 71(2):67-73. doi: 10.1016/j.recesp.2017.11.034

8. Nishimura R, Otto C, Bonow R, Carabello B, Erwin J3rd, Fleisher L, et al. 2017 AHA/ACC Focused update of the 2014 AHA/ACC guideline for the management of patients with valvular heart disease: a report of the American College of Cardiology/American Heart Association Task Force on clinical practice guidelines. Circulation 2017; 135(25):e1159-95. doi: 10.1161/CIR.0000000000000503

9. Yanagisawa R, Hayashida K, Yamada Y, Tanaka M, Yashima F, Inohara T, et al. Incidence, predictors, and mid-term outcomes of possible leaflet thrombosis after TAVR. JACC Cardiovasc Imaging 2016; S1936-878X(16)30897-X. doi: 10.1016/j.jcmg. 2016.11.005

10. Del Trigo M, Muñoz-García A, Latib A, Auffret V, Wijeysundera $H$, Nombela-Franco $L$, et al. Impact of anticoagulation therapy on valve haemodynamic deterioration following transcatheter aortic valve replacement. Heart 2018; 104(10):81420. doi: 10.1136/heartjnl-2017-312514

11. Hansson N, Grove E, Andersen H, Leipsic J, Mathiassen O, Jensen J, et al. Transcatheter aortic valve thrombosis: incidence, predisposing factors, and clinical implications. J Am Coll Cardiol. 2016; 68(19):2059-69. doi: 10.1016/j.jacc.2016.08.010

12. Jose J, Sulimov D, El-Mawardy M, Sato T, Allali A, Holy E, et al. Clinical bioprosthetic heart valve thrombosis after transcatheter aortic valve replacement: incidence, characteristics, and treatment outcomes. JACC Cardiovasc Interv. 2017; 10(7):686-7. doi: $10.1016 /$ j.jcin.2017.01.045

13. Brennan J, Edwards F, Zhao Y, O'Brien S, Booth M, Dokholyan R, et al. Early anticoagulation of bioprosthetic aortic valves in older patients: results from the Society of Thoracic Surgeons Adult Cardiac Surgery National Database. J Am Coll Car- 
diol. 2012; 60(11):971-7. doi: 10.1016/j.jacc.2012.05 029

14. ElBardissi A, DiBardino D, Chen F, Yamashita M, Cohn L. Is early antithrombotic therapy necessary in patients with bioprosthetic aortic valves in normal sinus rhythm? J Thorac Cardiovasc Surg. 2010; 139(5):1137-45. doi: 10.1016/j.jtcvs.2009. 10.064

15. Aceves-Velázquez E, Vieyra-Herrera G, Rodríguez-Chávez L. Evaluación de la estrategia de terapia antitrombótica posterior al cambio valvular aórtico con válvulas biológicas en pacientes de bajo riesgo trombótico. Arch Cardiol Méx. 2017; 88(5):339-46. doi: 10.1016/j.acmx.2017.06.009

16. di Marco F, Grendene S, Feltrin G, Meneghetti D, Gerosa G. Antiplatelet therapy in patients receiving aortic bioprostheses: a report of clinical and instrumental safety. J Thorac Cardiovasc Surg. 2007; 133(6):1597-603. doi: 10.1016/j.jtcvs.2006.12.060

17. Gherli T, Colli A, Fragnito C, Nicolini F, Borrello B, Saccani S, et al. Comparing warfarin with aspirin after biological aortic valve replacement: a prospective study. Circulation 2004; 110(5):496-500. doi: 10.1161/01.cir.0000137122.95108.52

18. Al-Atassi T, Lam K, Forgie M, Boodhwani M, Rubens F, Hendry P, et al. Cerebral microembolization after bioprosthetic aortic valve replacement: comparison of warfarin plus aspirin versus aspirin only. Circulation 2012; 126(11 Suppl 1):S239-44. doi: 10.1161/CIRCULATIONAHA.111.084772

19. Colli A, Mestres C, Castella M, Gherli T. Comparing warfarin to aspirin (WoA) after aortic valve replacement with the St. Jude Medical Epic heart valve bioprosthesis: results of the WoA Epic pilot trial. J Heart Valve Dis. 2007; 16(6):667-71.

20. Rafiq S, Steinbrüchel D, Lilleør N, Møller C, Lund J, Thiis J, et al. Antithrombotic therapy after bioprosthetic aortic valve implantation: Warfarin versus aspirin, a randomized controlled trial. Thromb Res. 2017; 150:104-10. doi: 10.1016/j.thromres.2016.11.021

21. De Marchena E, Mesa J, Pomenti S, Marin Y Kall C, Marincic X, Yahagi K, et al. Thrombus formation following transcatheter aortic valve repla- cement. JACC Cardiovasc Interv. 2015; 8(5):728-39. doi: 10.1016/j.jcin.2015.03.005

22. Heras M, Chesebro J, Fuster V, Penny W, Grill D, Bailey K, et al. High risk of thromboemboli early after bioprosthetic cardiac valve replacement. J Am Coll Cardiol. 1995; 25(5):1111-9. doi: 10.1016/ 0735-1097(94)00563-6

23. Carnicelli A, O'Gara P, Giugliano R. Anticoagulation after heart valve replacement or transcatheter valve implantation. Am J Cardiol. 2016; 118(9):141926. doi: 10.1016/j.amjcard.2016.07.048

24. Moinuddeen K, Quin J, Shaw R, Dewar M, Tellides G, Kopf G, et al. Anticoagulation is unnecessary after biological aortic valve replacement. Circulation 1998; 98(19 Suppl):II95-8; discussion II98-9.

25. Sundt T, Zehr K, Dearani J, Daly R, Mullany C, McGregor C, et al. Is early anticoagulation with warfarin necessary after bioprosthetic aortic valve replacement? J Thorac Cardiovasc Surg. 2005; 129(5):1024-31. doi: 10.1016/j.jtcvs.2004.11.028

26. Mérie C, Køber L, Skov Olsen P, Andersson C, Gislason G, Skov Jensen J, et al. Association of warfarin therapy duration after bioprosthetic aortic valve replacement with risk of mortality, thromboembolic complications, and bleeding. JAMA 2012; 308(20):2118-25. doi: 10.1001/jama.2012.54506

27. Riaz H, Alansari S, Khan M, Riaz T, Raza S, Luni F, et al. Safety and use of anticoagulation after aortic valve replacement with bioprostheses: a meta-analysis. Circ Cardiovasc Qual Outcomes 2016; 9(3):294-302. doi: 10.1161/CIRCOUTCOMES.115.002696

28. Leetmaa T, Hansson N, Leipsic J, Jensen K, Poulsen S, Andersen H, et al. Early aortic transcatheter heart valve thrombosis: diagnostic value of contrast-enhanced multidetector computed tomography. Circ Cardiovasc Interv. 2015; 8(4):e001596. doi: 10.1161/CIRCINTERVENTIONS.114.001596

29. Nakatani S. Subclinical leaflet thrombosis after transcatheter aortic valve implantation. Heart 2017; 103(24):1942-46. doi: 10.1136/heartjnl-2017-311818

30. Jilaihawi H, Asch F, Manasse E, Ruiz C, Jelnin V, Kashif M, et al. Systematic CT methodology for the evaluation of subclinical leaflet thrombosis. JACC Cardiovasc Imaging 2017; 10(4):461-70. doi: 10.1016/j.jcmg.2017.02.005 